Supplementary information for

\title{
Ultrafast Optical Modulation of Harmonic Generation in Two-Dimensional Materials
}

\author{
Yang Cheng, Hao Hong, Hui Zhao, Chunchun Wu, Yu Pan, Can Liu, Yonggang Zuo, Zhihong \\ Zhang, Jin Xie, Jinhuan Wang, Dapeng Yu, Yu Ye, Sheng Meng and Kaihui Liu
}

The supplementary information includes:

Supplementary Fig. 1-8 

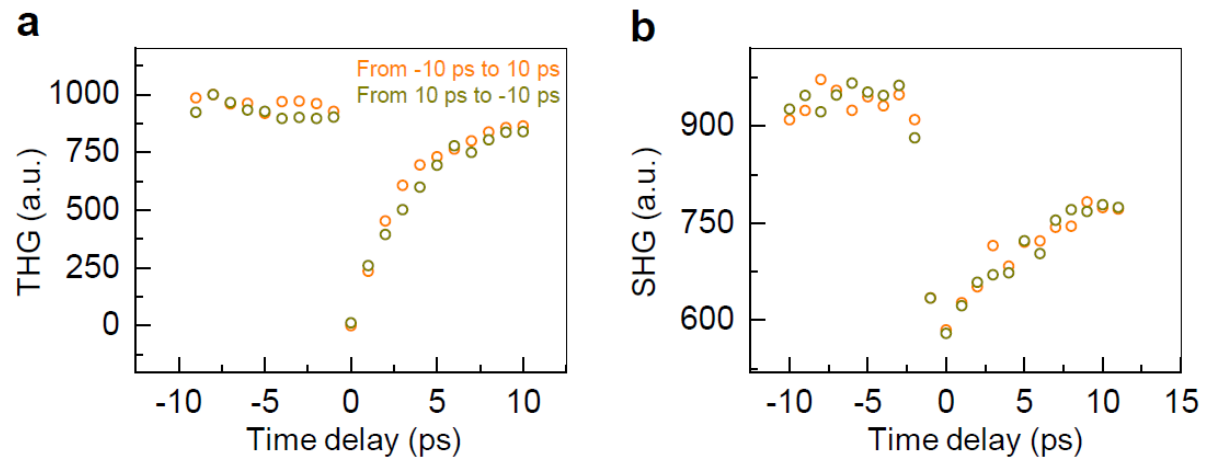

Supplementary Fig. 1 | Reversible all-optical modulation on harmonic generation of $\mathrm{MoS}_{2}$ and graphene monolayer. a, Time-resolved THG intensity of graphene monolayer with $3.1 \mathrm{eV}$ pump (under fluence of $38 \mathrm{~mJ} / \mathrm{cm}^{2}$ ) and $0.92 \mathrm{eV}$ probe (under fluence of $1.6 \mathrm{~mJ} / \mathrm{cm}^{2}$ ) from $-10 \mathrm{ps}$ to $10 \mathrm{ps}$ (orange circle) and from $10 \mathrm{ps}$ to $-10 \mathrm{ps}$ (dark green circle), respectively. $\mathbf{b}$, Time -resolved SHG intensity of $\mathrm{MoS}_{2}$ monolayer with $3.1 \mathrm{eV}$ pump (under fluence of $14 \mathrm{~mJ} / \mathrm{cm}^{2}$ ) and $0.92 \mathrm{eV}$ (under fluence of $0.5 \mathrm{~mJ} / \mathrm{cm}^{2}$ ) probe from $-10 \mathrm{ps}$ to $10 \mathrm{ps}$ (orange circle) and from $10 \mathrm{ps}$ to $-10 \mathrm{ps}$ (dark green circle), respectively. We can exclude the possibility of optical damage in samples due to the reversible results. 


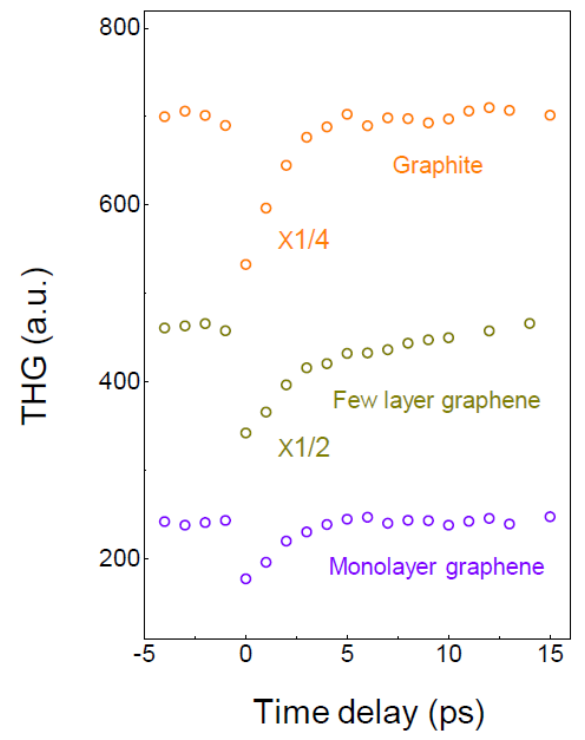

Supplementary Fig. 2 | Comparisons between THG response with all-optical modulation of graphite, few-layer graphene and graphene monolayer. The open circles show the evolution of THG intensity with all-optical modulation from graphite (orange circle), few-layer graphene (dark green circle), graphene monolayer (purple circle). These measurements are conducted using the same setup and conditions mentioned in Figure 2 of the main text ( $\hbar \omega_{1}=3.1 \mathrm{eV}$ of pump pulse, under fluence of $4 \mathrm{~mJ} / \mathrm{cm}^{2} ; \hbar \omega_{2}=0.92 \mathrm{eV}$ of probe pulse, under fluence of $1.6 \mathrm{~mJ} / \mathrm{cm}^{2}$ ). 
a

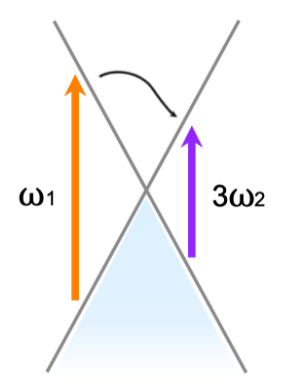

b

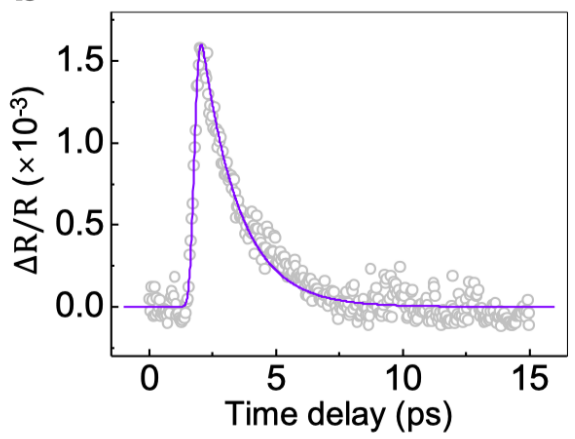

Supplementary Fig. 3 | Transient absorption signal of graphene monolayer. a, Scheme of the optical pump-probe measurements. b, The transient absorption spectrum is measured with $3.1 \mathrm{eV}$ pump (under fluence of $0.5 \mathrm{~mJ} / \mathrm{cm}^{2}$ ) and $2.4 \mathrm{eV}$ probe (under fluence of $2.2 \mathrm{~mJ} / \mathrm{cm}^{2}$ ). Noted that, the photon energy of probe beam is slightly smaller than $3 \hbar \omega_{2}=2.76 \mathrm{eV}$ of THG restricted by our experimental setup. The dynamic process of time-resolved relative changes in THG is very similar to the carrier dynamics in graphene monolayer which has the same rise-up and decay time. 

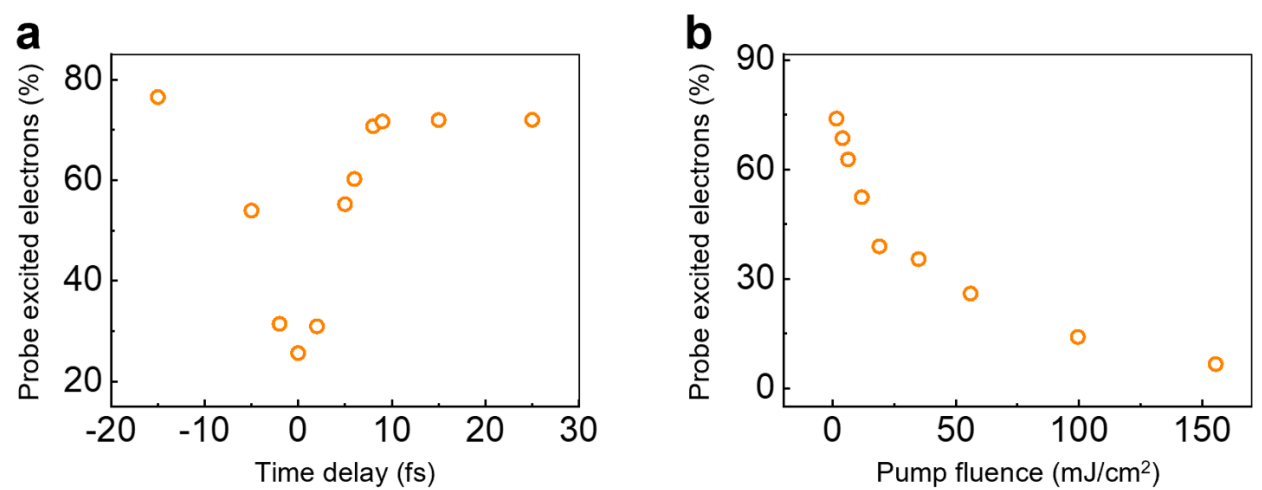

Supplementary Fig. 4 | Theory simulations of probe excited electrons with various time-delay and pump fluence. a, The proportion of probe excited electrons in energy bands with various time delay. The pump fluence is set as $56 \mathrm{~mJ} / \mathrm{cm}^{2}$, and the pulse width $2 \sigma$ is also set as 8 fs to simplify the calculations. $\mathbf{b}$, The proportion of probe excited electrons in energy bands with various pump fluence at $\Delta \mathrm{t} \approx 0$ fs. The tendency of the probe excited electrons evolving with the time delay and pump fluence is very similar to the results in Figure 3 of the main text. 

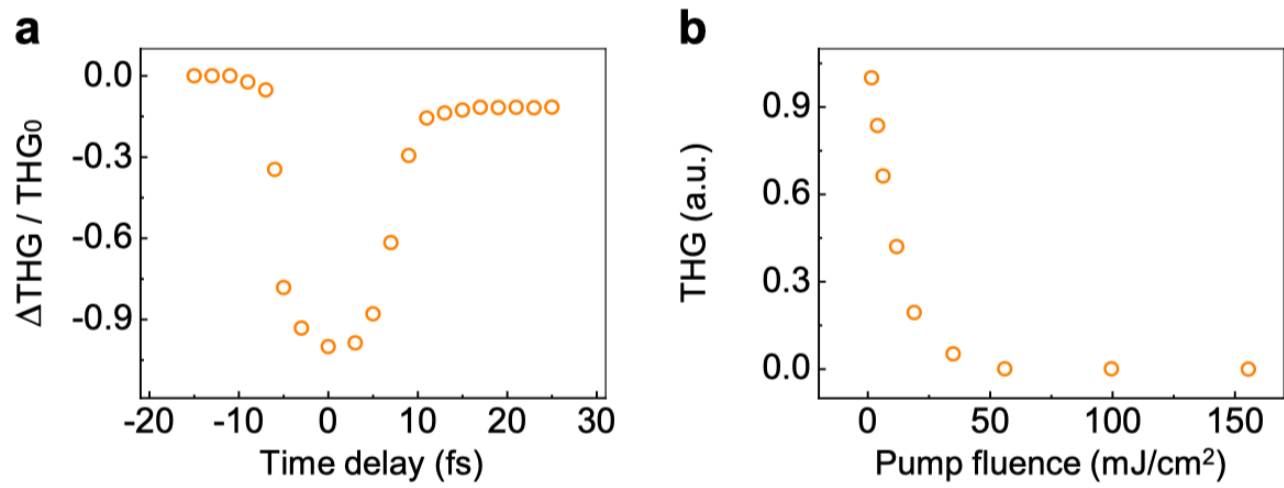

\section{Supplementary Fig. 5 | Theoretical simulations of all-optical modulation on THG from}

graphene monolayer with $1.55 \mathrm{eV}$ pump. a, Evolution of relative changes in THG intensity from the graphene monolayer under different time delay between $1.55 \mathrm{eV}$ pump and $0.92 \mathrm{eV}$ probe. $\mathbf{b}$, Simulative THG intensity variation with different pump fluence. The THG intensity decreases monotonously with increasing pump fluence. 

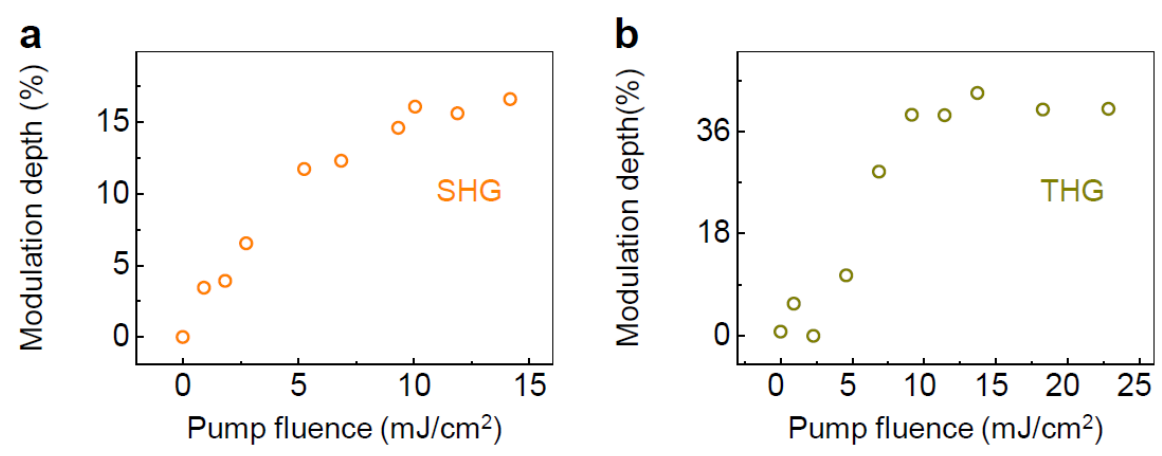

Supplementary Fig. $6 \mid$ Fluence dependence of the SHG and THG intensity of MoS monolayer with $3.1 \mathrm{eV}$ pump. a,b, Experimental relative changes in SHG and THG intensity of $\mathrm{MoS}_{2}$ monolayer with different pump fluence, respectively. The pump fluence of probe pulse is the same as in Figure 4. The modulation depth of $\mathrm{MoS}_{2}$ is about 16\% (43\%) for SHG (THG) process. 

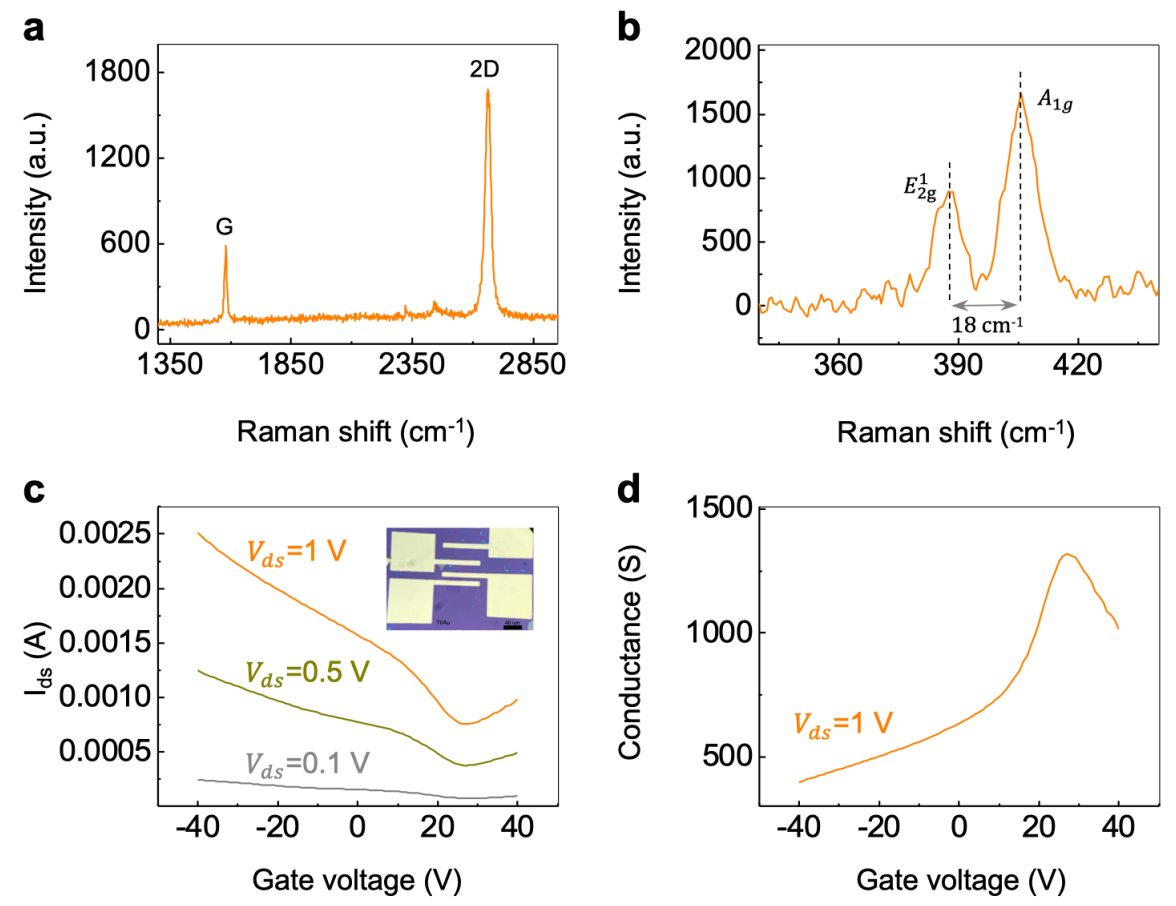

Supplementary Fig. 7 | Raman spectra of graphene and $\mathrm{MoS}_{2}$ monolayer. a, Raman spectrum of graphene monolayer without the D band, indicating the high quality of the graphene film. $\mathbf{b}$, Raman spectrum of $\mathrm{MoS}_{2}$ monolayer. The characteristic in-plane $E_{2 g}^{1}$ and out-of -plane $A_{1 g}$ phonon modes are labelled. The $18 \mathrm{~cm}^{-1}$ separation between these two peaks $\left(\omega_{A}-\omega_{E}\right)$ indicates that our $\mathrm{MoS}_{2}$ samples are monolayers in nature. $\mathbf{c}, I_{\mathrm{ds}}$ as a function of back-gate voltages $\left(V_{\mathrm{G}}\right)$ at various $\mathrm{V}_{\mathrm{ds}}$. The inset shows the optical microscopy image of our device. Scale bar: $40 \mu \mathrm{m}$. d, Conductance as a function of back-gate voltages $\left(\mathrm{V}_{\mathrm{G}}\right)$ at $\mathrm{V}_{\mathrm{ds}}=1 \mathrm{~V}$. We can deduce that the $\mathrm{V}_{\text {Dirac }}$ is at $27 \mathrm{~V}$. 


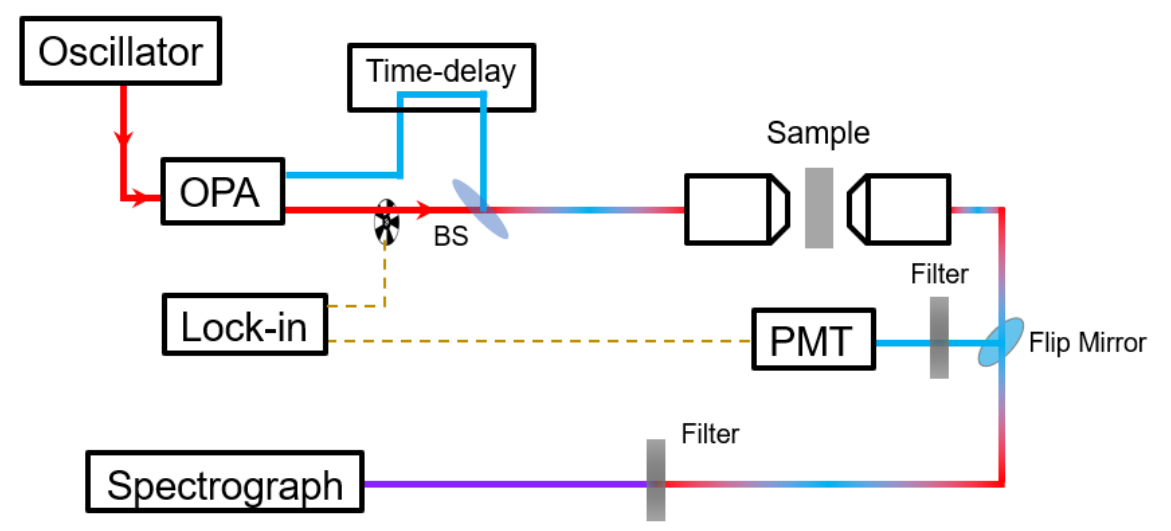

Supplementary Fig. 8 | Scheme of the optical pump-probe measurements set-up. The timeresolved THG and SHG is excited with femtosecond pulses (Coherent laser, $150 \mathrm{fs}, 250 \mathrm{KHz}$ ) generated by a Ti:sapphire oscillator series pumping an OPA. PMT: Photomultiplier. 